OPEN ACCESS

Edited by:

Guohua Chai,

Qingdao Agricultural University, China

Reviewed by:

Jianhua Wei,

Beijing Academy of Agricultural and Forestry Sciences, China

Lin Zhang,

Central South University Forestry and

Technology, China

${ }^{*}$ Correspondence:

Fang Tang

tangfangcaf@126.com

Specialty section:

This article was submitted to

Plant Biotechnology,

a section of the journal

Frontiers in Plant Science

Received: 26 May 2021

Accepted: 21 June 2021

Published: 14 July 2021

Citation:

Chu L, He X, Shu W, Wang L and Tang F (2021) Knockdown of miR393

Promotes the Growth and Biomass

Production in Poplar.

Front. Plant Sci. 12:714907. doi: 10.3389/fpls.2021.714907

\section{Knockdown of miR393 Promotes the Growth and Biomass Production in Poplar}

\author{
Liwei Chu ${ }^{1}$, Xuejiao $\mathrm{He}^{1}$, Wenbo Shu ${ }^{3}$, Lijuan Wang ${ }^{1,2}$ and Fang Tang ${ }^{1,2 *}$ \\ 'State Key Laboratory of Tree Genetics and Breeding, Key Laboratory of Tree Breeding and Cultivation of the National \\ Forestry and Grassland Administration, Research Institute of Forestry, Chinese Academy of Forestry, Beijing, China, \\ ${ }^{2}$ Co-innovation Center for Sustainable Forestry in Southern China, Nanjing Forestry University, Nanjing, China, ${ }^{3}$ Key \\ Laboratory of Horticultural Plant Biology of Ministry of Education, College of Horticulture and Forestry Sciences, Huazhong \\ Agricultural University, Wuhan, China
}

Short tandem target mimic (STTM), which is composed of two short sequences mimicking small RNA target sites, separated by a linker of optimal size, can block the functions of all members in a miRNA family. microRNA393 (miR393), which is one of the conserved miRNA families in plants, can regulate plant root growth, leaf development, plant architecture, and stress resistance. In order to verify the role of miR393 in the secondary growth of trees, we created its STTM transgenic poplar lines (STTM393). The expression of miR393 in STM393 lines was reduced by over 10 times compared with the control plants. STTM393 lines showed promoted growth with about 20\% higher, 15\% thicker, and 2-4 more internodes than the control plants after 3 months of growth. The cross-section of the stems showed that STTM393 lines had wider phloem, xylem, and more cambium cell layers than control plants, and the lignin content in STTM393 lines was also higher as revealed by staining and chemical determination. Based on the transcriptome analysis, the genes related to the auxin signaling pathway, cell cyclin, cell expansion, and lignin synthesis had higher expression in STTM393 lines than that in control plants. The higher expression levels of $F B L$ family members suggested that the auxin signaling pathway was strengthened in STTM393 lines to promote plant growth. Therefore, the knockdown of miR393 using the STTM approach provides a way to improve poplar growth and biomass production.

Keywords: miR393, STTM, plant growth, auxin signaling pathway, RNA-seq, poplar

\section{INTRODUCTION}

Poplar is one of the important carbon-neutral biomass for the production of timber products, paper pulping, chemicals, and biofuels (Gui et al., 2020). At present, there is a large demand for wood, and cultivating fast-growing trees like poplar is a promising way to solve this problem. To further improve poplar growth and biomass production, substantial efforts have been made to identify the functions of genes and small RNAs that regulate poplar growth (Lucas et al., 2013; Sundell et al., 2017; Busov, 2018). MicroRNAs (miRNAs) can negatively regulate the transcription and post-transcription of their specific target genes by binding their near-perfect complementary sites through its 20-24 nucleotides, which can lead to the target mRNA degradation and/or translational repression (Meyers et al., 2008; Voinnet, 2009). 
In previous studies, in order to understand miRNA functions, the major approaches were used by generating the transgenic lines in which the genes that encode miRNAs or the target gene of miRNAs were overexpressed (Zhu et al., 2009; Zhang et al., 2010; Curaba et al., 2013). However, these approaches were insufficient to fully understand miRNA functions. First, one miRNA often targeted multiple genes for regulation, and modifying one target gene could not possibly reveal the functions of other target genes (Bartel, 2009). Second, overexpressing the miRNAs could only result in a decrease in the expression level of miRNA target genes and an inability to obtain the information of gain of functions of the target genes. On the contrary, miRNAs tend to be gene families, and thus previous approaches could generally overexpress only one member at a time (Sieber et al., 2007; Curaba et al., 2013).

Short tandem target mimic (STTM) can explore the functions of miRNAs by simultaneously blocking all the members in an miRNA family so as to achieve a gain-function of target genes through a single genetic transformation event (Yan et al., 2012). STTM is an artificial, short ( $100 \mathrm{nt})$, non-coding RNA that is composed of two miRNA-binding sites and a spacer/linker. The two binding sites are complementary to the target small RNAs except for the central 3-nucleotide bulge, which sticks out between the 10th and 11th nucleotides of the targeted small RNA, so that the binding sites can trap miRNAs without being cleaved by them (Tang et al., 2012). STTM can be expressed through either stable plant transformation or virusbased transient expression systems (Sha et al., 2014; Teotia et al., 2017) and has been used to knockdown the target miRNA expression in Arabidopsis (Yan et al., 2012), soybean (Wong et al., 2014), tomato (Cao et al., 2016), cotton (Gu et al., 2014), and maize (Liu et al., 2019).

As one of the conserved miRNA families in plants, miR393 had been studied in several species like Arabidopsis (Navarro et al., 2006), rice (Bian et al., 2012), barley (Yuan et al., 2019), and poplar (Lu et al., 2008), and it was found that miR393 affected the plant root system (Vidal et al., 2010), leaf development (Si-Ammour et al., 2011), seedling growth and drought stress tolerance (Yuan et al., 2019), and salinity and alkaline stress (Gao et al., 2011). In Arabidopsis, miR393, which is encoded by MIR393a and MIR393b, can regulate its target genes including four F-box family auxin receptor genes (Transport Inhibitor Response Protein 1, TIR1) by splicing (Zhang et al., 2006; SiAmmour et al., 2011; Wójcik and Gaj, 2016). TIR1 family members affect different phenotypes including rooting, seedling growth, and seed coat development by regulating the auxin pathway (Parry et al., 2009; Hu et al., 2012). In poplar, the $F B L$ family, which are homologous genes of the TIR family in Arabidopsis, have eight members, and FBL1-FBL4 have been confirmed to be cleaved by miR393 (Shu et al., 2015; Tang et al., 2020). There are four members in the miR393 family of poplar, in which the mature miR393 encoded by different precursors share the same sequences (PmiREN, V2) (Guo et al., 2019). In a previous study, we analyzed the expression of miR393 in different tissues of poplar at multiple developmental stages and found that its expression was low in young tissues but high in mature tissues, especially in old stem (Tang et al., 2020). This suggests that miR393 may play a role in the vascular tissue development, which deserves further investigation.

In this study, we created miR393 STTM transgenic poplar (Populus alba $\times$ Populus glandulosa) in which the expression of miR393 was significantly reduced. The transgenic lines showed increased plant height, ground diameter, number of internodes, width of xylem, and total biomass production compared with the non-transgenic control plants. Accordingly, a large number of genes related to plant growth and wood formation were upregulated in STTM393 transgenic lines in order to inhibit the expression of miR393. The results support that blocking the function of miR393 by the STTM approach can promote plant growth and increase biomass production.

\section{MATERIALS AND METHODS STTM393 Vector Construction and Transformation}

The construction of the STTM393 vector was based on the previous method (Tang et al., 2012; Peng et al., 2018). Two primers with the sequences to form hairpin were synthesized according to the sequence of mature miR393, which is conserved across all the sequenced poplar species so far. The primer sequences were as follows: STTM393-Forward Primer: 5'gccATTTAAATatggtctaaagaagaaggatGATCAATGCGAtcgTCC CTTTGGAgaattcggtacgctgaaatcaccag- $3^{\prime}$ and STTM393-Reverse

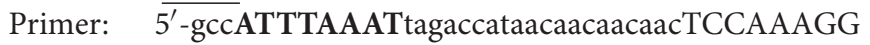
GAcgaTCGCATTGATCaagcttgggctgtcctctccaaatg-3'. The SwaI site "ATTTAAAT," the EcoRI site "gaattc," the HindII site "aagctt," the artificial linker (20 nts) with the lowercase nucleotides, the "tcg" bulge in the middle of miR393 sequences, and the complementary sequences to $\mathrm{d} 35 \mathrm{~S}$ and $\mathrm{T}-35 \mathrm{~S}$ of the pOT2 vector with lowercase nucleotides at the $3^{\prime}$-end were embedded in these two synthesized primers. Then, the pOT2 vector was used as a template for PCR amplification, and the linker PCR product was subjected to SwaI digestion and a subsequent self-ligation, which resulted in pOT2-STTM393. Finally, the pOT2 with STTM393 structure was sub-cloned into a binary vector pFGC5941, which was used to transform into $84 \mathrm{~K}$ poplar $(P$. alba $\times$ P. glandulosa) by Agrobacterium-mediated leaf disk method. The positively transformed lines identified by resistance screening and expression analysis were used for further phenotypic observation.

\section{Plant Phenotypic Determination and Chemical Composition Measurement}

The plantlets of STTM393 transgenic lines and 84K controls were grown in the culture medium for 1 month and then transferred to the soil pots. Seedlings were grown in a culture room under

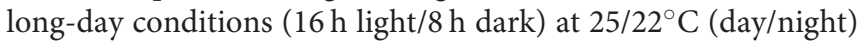
and in a greenhouse under natural light with daytime and nighttime temperatures of $24-30^{\circ} \mathrm{C}$ in the Chinese Academy of Forestry (Beijing, China). The plant height, ground diameter, and internode number were measured every month. The plant height is the length from the top of the plant to the base of the stem; the ground diameter is the diameter of the last internode at the 
base of the stem, and the number of internodes is the internode from the first expanded leaf to the last internode at the stem base. Stem tissues were sectioned by Leica VT1000S vibratingblade microtome (Leica, Germany) with a thickness of $70 \mu \mathrm{m}$. The sections were stained by using toluidine blue (TBO) and phloroglucinol- $\mathrm{HCl}$ and were observed under Olympus BX51 microscope (Olympus, Tokyo, Japan). The stems of 6-month-old plants from 16th to 40th were used for cellulose, hemicellulose, and lignin content determination, according to the published protocol (Wang et al., 2020). Six STTM393 lines and 84K control plants were analyzed in this study, and each line included 610 vegetatively propagated individuals for the experiment. Data from each experiment were subjected to either an ANOVA or a $t$-test. All statistical analyses were performed using the SPSS Version 17.0 (SPSS, NY, United States). The phenotyping of STTM393 transgenic lines and $84 \mathrm{~K}$ control plants was performed on three batches of plants.

\section{Total RNA Extraction and cDNA Synthesis}

Total RNA was extracted using the LC Sciences Total RNA Purification Kit (\#TRK-1001, LC Sciences, TX, United States) according to the previous modified methods (Tang et al., 2019). The integrity of total RNA was further assessed by $1.5 \%$ agarose gel electrophoresis, and the RNA concentration and purity were determined by NanoDrop ${ }^{\mathrm{TM}} 8000$ Spectrophotometer (Thermo Fisher Scientific, MA, United States). Only RNA samples with an A260/A280 ratio between 1.9 and 2.1 and an A260/A230 ratio $>1.80$ were used for $\mathrm{CDNA}$ synthesis. We used a universal reverse transcription (RT) PCR method, in which the total RNA will be added by a poly(A) tail before RT (Tang et al., 2016). First, $2 \mu \mathrm{g}$ of the total RNA was polyadenylated with ATP by poly(A) polymerase (PAP) at $37^{\circ} \mathrm{C}$ for $1 \mathrm{~h}$ in a 20 $\mu l$ reaction mixture using the Poly(A) Tailing Kit (\#AM1350, Invitrogen, MA, United States). Then, $10 \mu \mathrm{l}(1 \mu \mathrm{g})$ of the E-PAPtreated total RNA was reverse transcribed with a poly(T) adapter universal RT-primer (5'-AAC GAG ACG ACG ACA GAC TTT TTT TTT TTT TTTV-3') using PrimeScript ${ }^{\mathrm{TM}}$ RT reagent Kit (\#RR037, TaKaRa, Shiga, Japan) following the instruction of the manufacturer. The cDNA was diluted 20 -fold with nuclease-free water for quantitative real-time reverse transcription PCR (qRTPCR).

\section{Quantitative Real-Time PCR Analysis}

Quantitative real-time PCR was performed on the LightCycler ${ }^{\circledR}$ 480 System (Roche Molecular Systems, Basel, Switzerland). The reaction mixture contained $10 \mu \mathrm{l}$ of KAPA SYBR FAST qPCR Master Mix (\# K4601, KAPA Biosystems, MA, United States), $2 \mu \mathrm{l}$ of 20 -fold diluted cDNA, $0.4 \mu \mathrm{M}$ each of a forward and a reverse primer (Supplementary Table 1), and $\mathrm{ddH}_{2} \mathrm{O}$ in a final volume of $20 \mu \mathrm{l}$. Amplifications were performed with the following program: $95^{\circ} \mathrm{C}$ for $3 \mathrm{~s} ; 40$ cycles of $95^{\circ} \mathrm{C}$ for $10 \mathrm{~s}, 60^{\circ} \mathrm{C}$ for $30 \mathrm{~s}$, and $72^{\circ} \mathrm{C}$ for $3 \mathrm{~s}$. No-template reactions were used as negative controls, and protein phosphatase 2A-2 (PP2A-2) and polyubiquitin $(U B Q)$ were used as reference genes (Tang et al., 2019). Each sample was assessed in four technical replicates, and the experiment was repeated three times.

\section{High-Throughput Transcriptome Sequencing}

The top five internodes of 3-month-old STTM393 lines (STTM393-2, STTM393-8, and STTM393-16) and 84K control plants were collected, among which 3-5 individuals in a line were randomly selected as a biological replicate, and each line had three biological replicates. The cDNA library construction and high-throughput sequencing of the above samples were performed by Biomarker Technologies (BMK, Beijing, China). The sequencing library used the NEBNext Ultra ${ }^{\mathrm{TM}}$ RNA Library Prep Kit for Illumina (NEB, MA, United States). The library was sequenced with Illumina Novaseq 6000, and the reading length was $2 \times 150 \mathrm{bp}$ (PE150). Clean data were obtained by removing the sequences containing adapter, poly- $\mathrm{N}$, and low quality from raw data. Using Hisat2 software, the obtained clean data were compared with the genome of 717 poplar (Populus tremula $\times$ P. alba) (http://128.192.158.63/index.php/databases/spta-717genome). The number of allowed mismatched nucleotides was set to "0" or "1." After that, String-Tie (version 2.1.4) was used for transcriptional assembly and new transcriptional prediction, and the alternative splicing types and corresponding expression levels of each sample were obtained by ASprofile software. The quantification of gene expression level was estimated by the number of fragments per kilobase of transcript per million fragments (FPKM). EBSeq (Version 1.5.4) software was used to analyze the differential expression between STTM393 lines and $84 \mathrm{~K}$ control samples. Genes with an adjusted FDR $<0.05$ found by DESeq2 were assigned as differentially expressed. Gene ontology (GO) enrichment analysis of differentially expressed genes (DEGs) was carried out by GOseq R software, and the DEGs in the KEGG pathway were enriched by KOBAS software. The original transcriptome data were submitted to the NCBI public database Sequence Read Archive (SRA, PRJNA724789).

\section{RESULTS}

\section{STTM393 Promotes Poplar Growth}

We obtained 30 STTM393 transgenic lines by Agrobacteriummediated leaf disk method, and qRT-PCR showed that the expression of miR393 in 13 randomly selected lines was reduced over 10 times (Supplementary Figure 1). Six STTM393 transgenic lines with different expression levels were randomly selected for phenotype observation. The height, ground diameter, and internode number of these STTM393 transgenic plants at different developmental stages were significantly higher than those of $84 \mathrm{~K}$ control plants (Supplementary Figure 2). Three STTM393 lines (i.e., STTM393-2, STTM393-8, and STTM393-16) with obvious phenotypic changes were used for further analysis.

The transgenic plants of STTM393-2, STTM393-8, and STTM393-16 grew faster than the control plants from tissue culture to soil cultivation. The plant height and root length of their 1-month-old tissue culture plantlets were longer than the control plantlets (Supplementary Figure 3A). Similarly, the STTM393 transgenic plants transferred to soil for 1 month (2-month-old plants) had significantly higher plant 


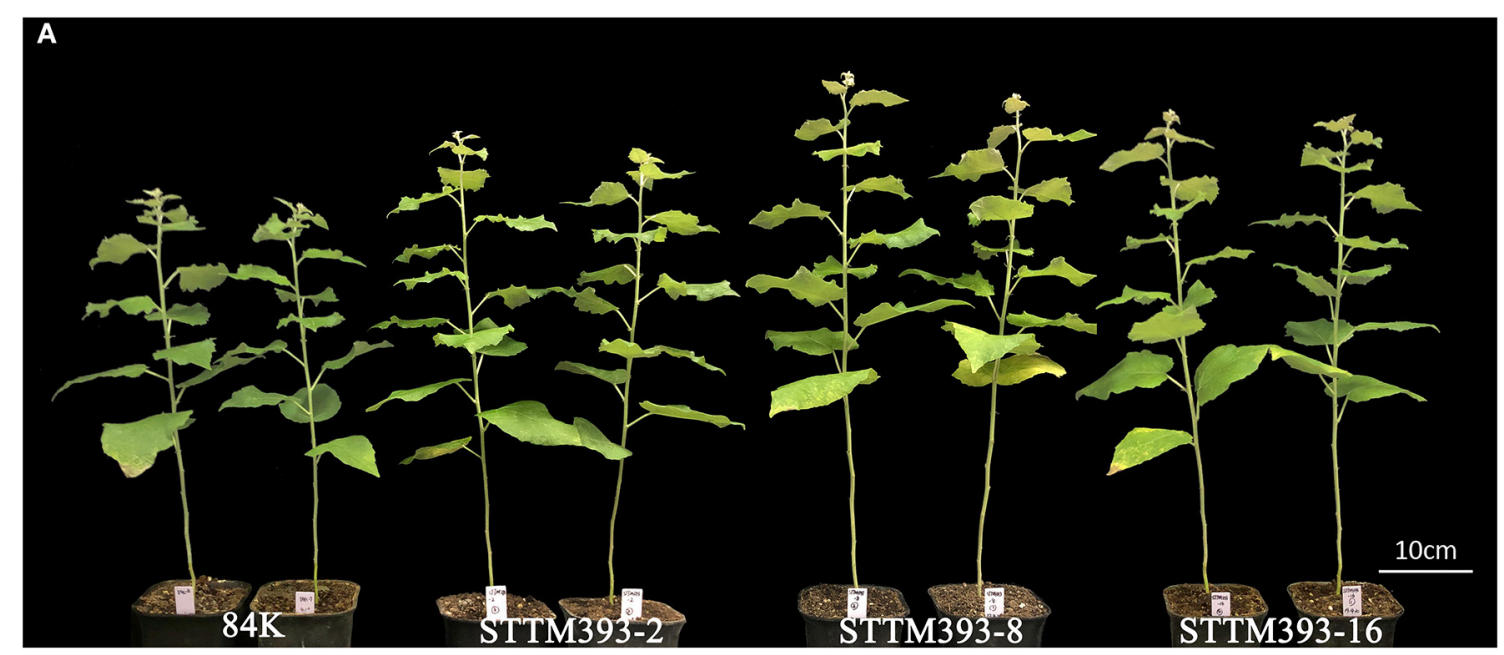

B
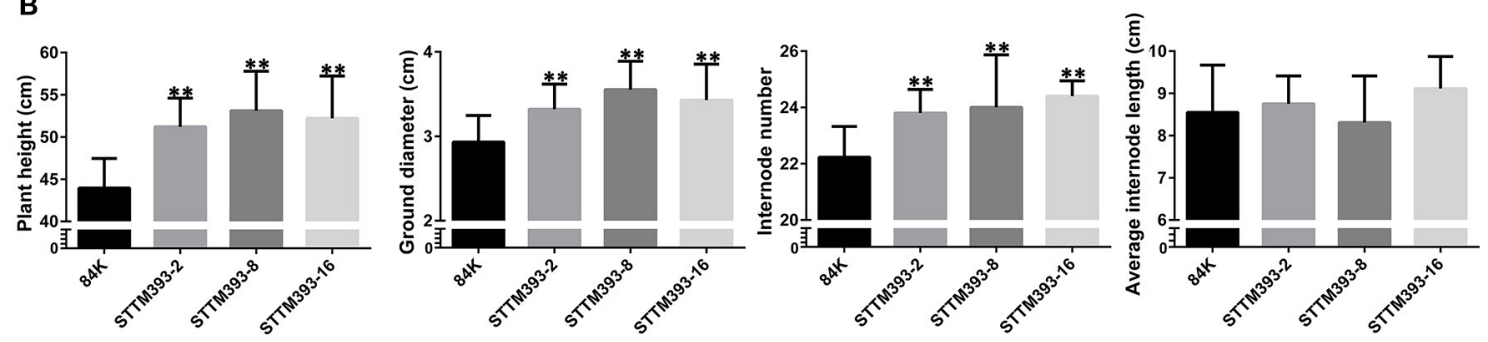

FIGURE 1 | The phenotypes of STTM393 transgenic plants. (A) 3-month-old STTM393 and 84K control plants. (B) The height, ground diameter, internode number, and average internode length of 3-month-old STTM393-2, STTM393-8, STTM393-16, and 84K control plants. The bars represent means \pm SD $(n=10)$, ${ }^{\star \star} P \leq 0.01$.

height, ground diameter, and internode number than those of the control plants (Supplementary Figures 3B-E). The height, ground diameter, and internode number of each 3-monthold STTM393 lines were 15-35, 15-25, and 7-13\% higher than those of the control, respectively. However, the average internode length of STTM393 plants was not significantly different from that of the control plants (Figure 1). The three transgenic lines were grown three times, and similar results were obtained (Supplementary Figure 4). Therefore, the knockdown of miR393 family may promote plant growth in poplar.

\section{STTM393 Enhances Wood Formation}

To investigate the function of miR393 in wood formation, we prepared the stem cross-sections to observe the vascular tissue of STTM393 lines. The results showed that the 15 th internode of 3-month-old STTM393 transgenic plants was significantly larger in diameter than that of the control plants, while the cell layers of cambium and the width of phloem, xylem, and pith were also larger than of the control plants (Figure 2). This indicates that STTM393 transgenic plants can enhance the xylem development probably due to the high activity of cambium cells.

Phloroglucinol staining showed that lignin deposition was increased in both the fiber and vessel cell walls of STTM393 transgenic lines, as revealed by the dark pink color (Figures 3A,B). Therefore, the content of lignin, cellulose, and hemicellulose in the stems of STTM393 transgenic lines and the controls was chemically determined. The STTM393 lines had significantly higher lignin content but lower cellulose content than the $84 \mathrm{~K}$ controls (Figure 3C). However, there was no significant difference in the relative hemicelluloses content between STTM393 transgenic and control plants (Figure 3C). This suggests that the inhibition of miR393 expression may increase the lignin content in the secondary cell wall.

\section{STTM393 Increases the Expression of PagFBLs}

We detected the expression levels of miR393 in the stems of 3-month-old STTM393 lines and 84K controls. The expression values of miR393 were reduced by 78,88 , and $91 \%$ in STTM393-2, STTM393-8, and STTM393-16 lines, respectively, compared with the control plants (Figure 4A). It indicates that the STTM of miR393 indeed causes the inhibition of the expression of miR393. FBL family members are the target genes of miR393 in poplar (Tang et al., 2020); thus, we detected PagFBLs expression in STTM393 lines and control plants by qRT-PCR and found that the expression levels of eight PagFBL genes were increased in STTM393 transgenic lines (Figure 4B). These results suggest that STTM393 can increase the expression of PagFBLs by inhibiting the function of miR393. 


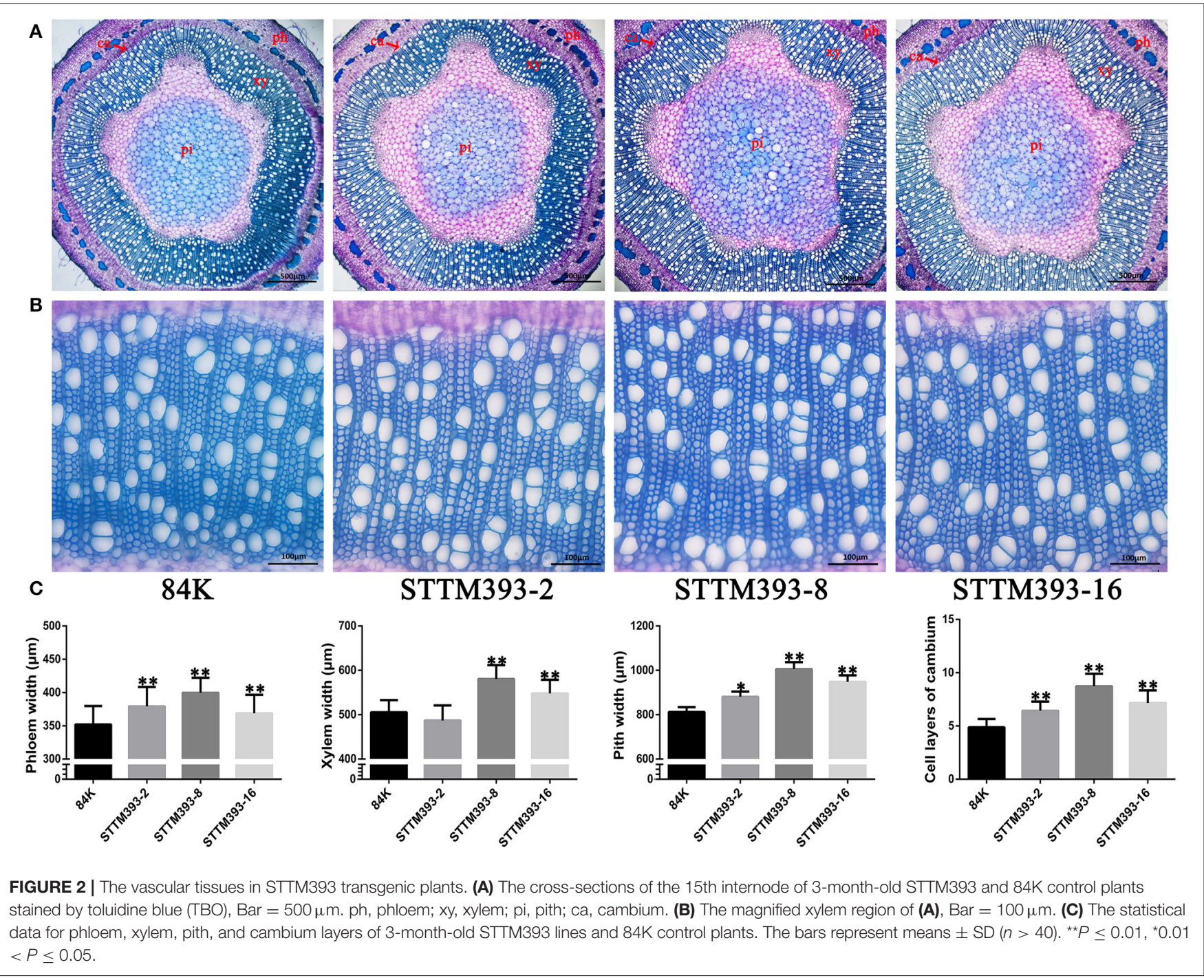

\section{Inhibition of miR393 Affects Genes Related to Plant Growth and Wood Formation}

To understand which genes would be affected due to the inhibited miR393 expression, the expression of genes in three STTM393 lines (STTM393-2, STTM393-8, and STTM393-16) and control plants was detected by RNA sequencing. The results showed that there were 863 differentially expressed genes in the three STTM393 lines compared with the control, 431 of which were upregulated, and 432 were downregulated (Supplementary Table 2). The biological function of the upregulated genes was mainly enriched in the xyloglucan metabolic process (GO:0010411), cell wall biogenesis (GO:0042546), cell wall organization (GO:0071555), and response to auxin (GO:0009733), while that of the downregulated genes was mainly enriched in response to stimulus (GO:0050896), response to stress (GO:0006950), cellular response to sulfur starvation (GO:0010438), and response to an inorganic substance (GO:0010035).
Notably, the expression of genes related to auxin signal transduction had been upregulated, such as auxin-responsive protein (SAUR1 and SAUR78), indole-3-acetic acid-amido synthetase (GH3.6), and indole-3-acetic acid-induced protein (ARG7). In addition, one auxin transporter protein, AUX1, and two homologous genes of the auxin-binding protein, $A B P 19 a$, had higher expression in STTM393 lines than in the control (Table 1). In STTM393 lines, several cell wall loosening factors including expansin (EXP), xyloglucan endotransglucosylase/hydrolase (XTH), glucan endo-1, 3-betaglucosidase $(G L C)$, and galacturonosyltransferase-like (GATL) were upregulated. Furthermore, the expression of cyclin genes regulating cell cycle transition was also upregulated, for example, cyclin D 1;1 (CYCD1;1), cyclin D 3;1 (CYCD3;1), and cyclin P $4 ; 1$ (CYCP4;1), all highly expressed in STTM393 transgenic lines. The higher expression of cell cycle-related genes may explain why the growth rate of STTM393 lines is faster than the controls.

In accordance with the relatively high content of lignin but low content of cellulose in STTM393 transgenic plants, the genes 

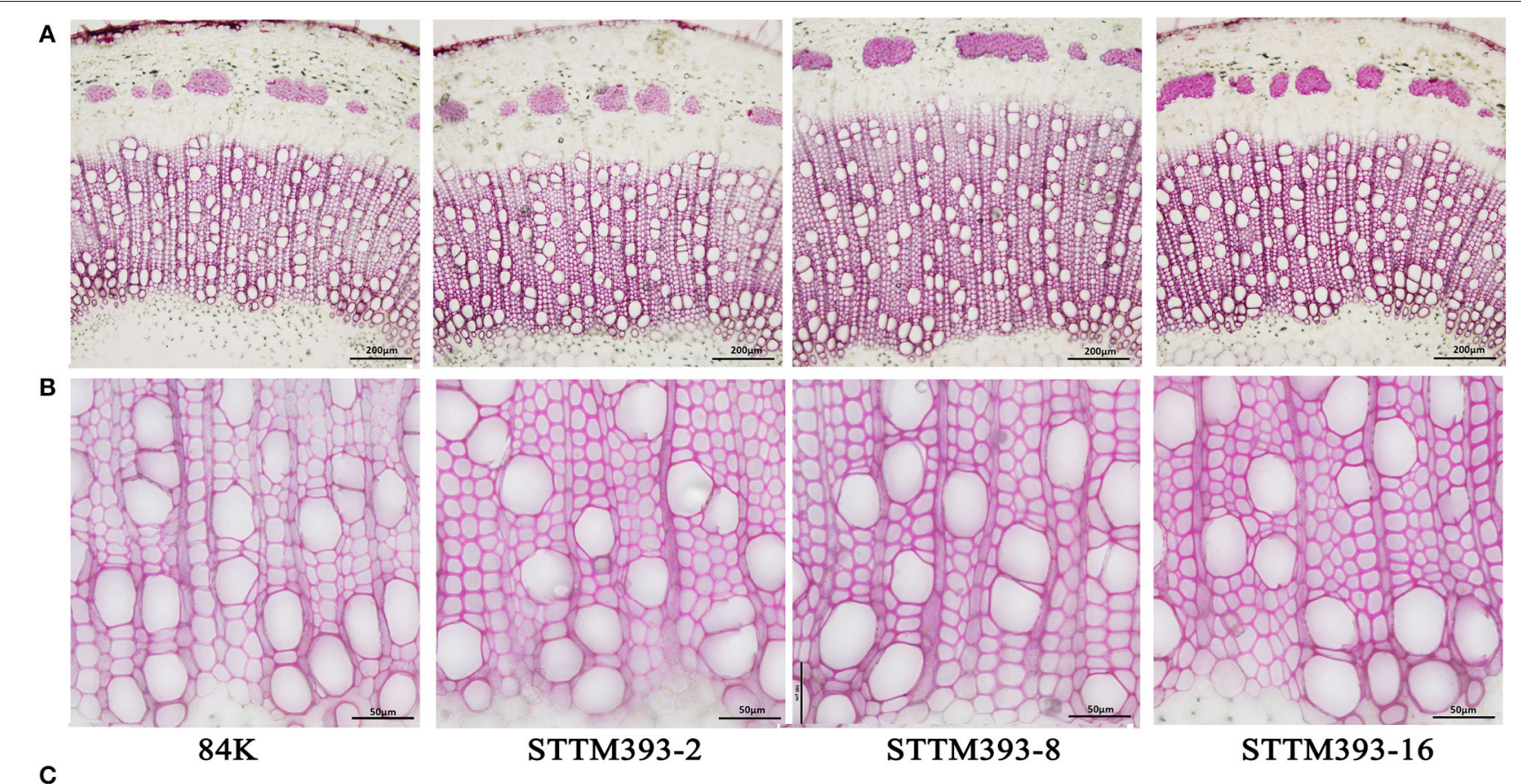

STTM393-2

STTM393-8
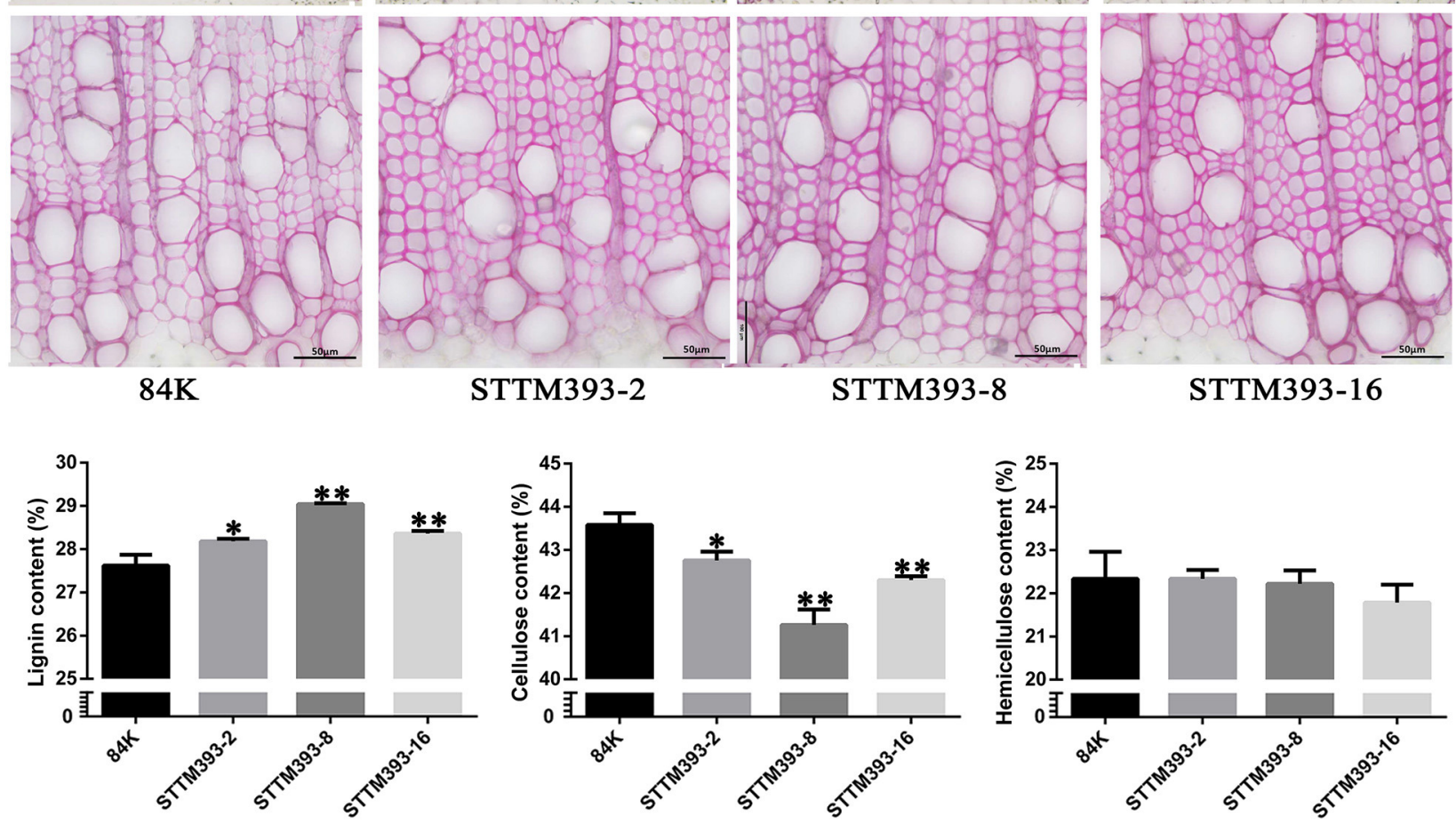

FIGURE 3 | The chemical composition analysis in STTM393 transgenic plants. (A) The cross-sections of the 15th internode of 6-month-old STTM393 and 84K control plants stained by phloroglucinol- $\mathrm{HCl}$, Bar $=200 \mu \mathrm{m}$. (B) The magnified xylem region of (A), Bar $=50 \mu \mathrm{m}$. (C) The lignin, cellulose, and hemicellulose content of stem from 16 th to 40 th internodes of 6 -month-old STTM393 lines and $84 \mathrm{~K}$ control plants. The bars represent means $\pm \mathrm{SD}(n>6)$. ${ }^{\star \star} P \leq 0.01$, ${ }^{\star} 0.01<P \leq 0.05$.

involved in the lignin synthesis pathway, such as cinnamoyl-CoA reductase (CCR1 and CCRL6), caffeic acid 3-O-methyltransferase (OMT1), peroxidase (PER9 and PER73), and benzylalcohol $O$-acetyltransferase $(B E A T)$, were upregulated, while cellulose synthase-like genes (CSLG3 and CSLE1) were downregulated (Table 1). The homologous genes of CESA1 and CESA6, which are involved in the cellulose synthesis in the primary cell wall, exhibited higher expression in STTM393 lines. Therefore, the inhibition of the miR393 expression can increase the expression of genes related to lignification, thus promoting wood formation.

The expression levels of some genes mentioned above were verified by qRT-PCR. PP2A-2 and UBQ were selected as reference genes for qRT-PCR. The expression trends of the genes calculated with PP2A-2 (Figure 5) were consistent with those calculated with $U B Q$ (Supplementary Figure 5). The relative expression levels of these genes obtained via qRT-PCR were consistent with that from the RNA sequencing data.

\section{DISCUSSION}

As an important regulator in the auxin signaling pathway, miR393 is involved in root development and stress resistance in herbaceous plants. For instance, the overexpression of miR393 can cause phenotypic changes in the length of main roots and the number of lateral roots in Arabidopsis and rice (Chen et al., 2011; Bian et al., 2012). We previously found that miR393 was highly expressed in developing secondary vascular tissues in poplar (Tang et al., 2016), but, so far, the role of miR393 in vascular tissue development has not been fully investigated. In this study, we blocked the miR393 expression in transgenic poplars using STTM technology and found its role in strengthening auxin signaling, which led to the promotion of secondary growth and production of high biomass in transgenic poplars.

To access the role of miR393 in secondary growth, we generated miR393 inhibition lines using the STTM approach. 


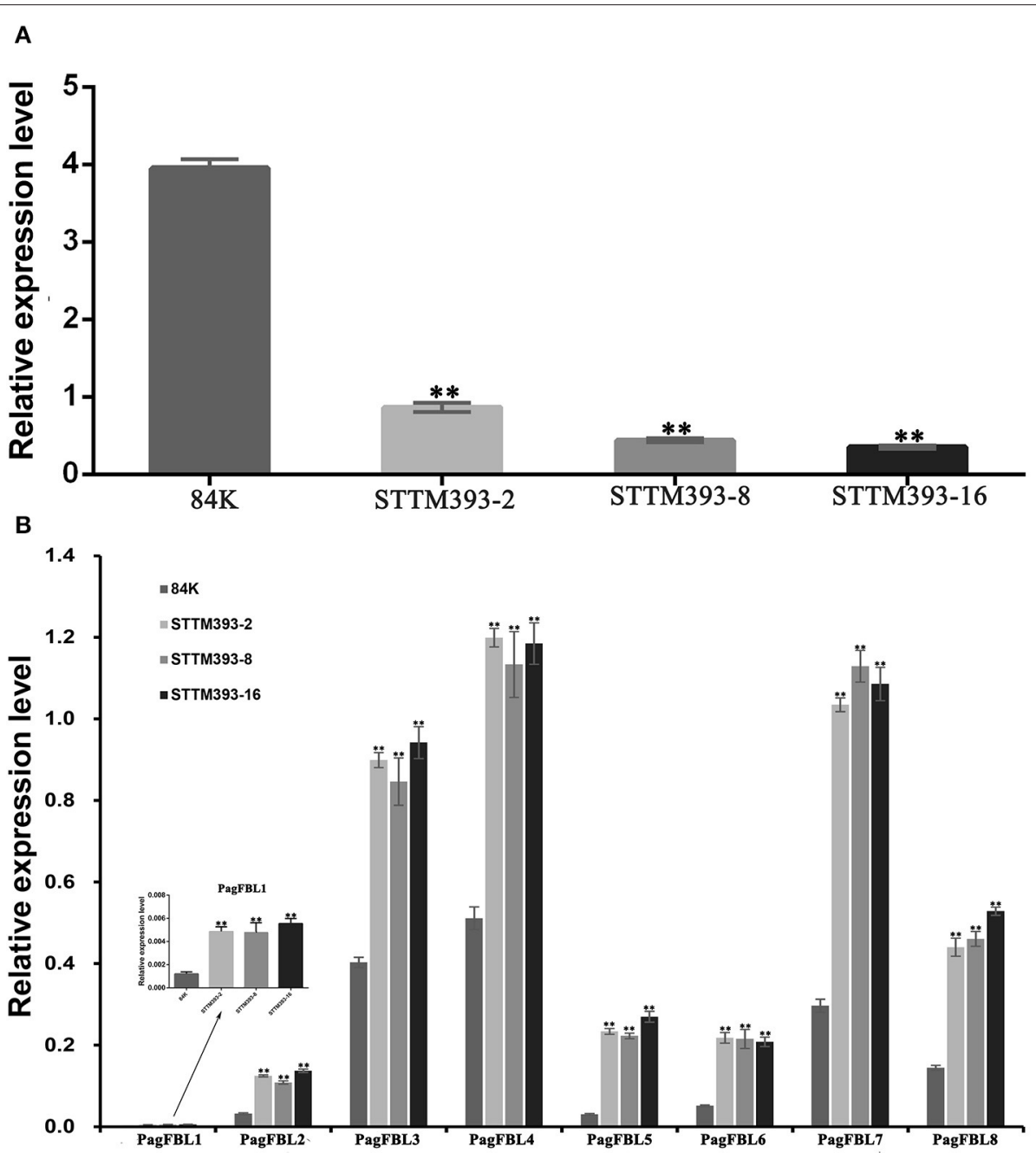

FIGURE 4 | The expression of miR393 and PagFBLs in STTM393 lines by quantitative real-time reverse transcription (qRT)-PCR. The relative expression of miR393 (A) and eight members from PagFBL family (B) was detected by qRT-PCR in the 3-month-old STTM393 lines and $84 \mathrm{~K}$ control plants. ${ }^{* \star} P \leq 0.01$.

The plant height, ground diameter, and the number of internodes of STTM393 transgenic lines were all increased significantly, and this was opposite to the phenotype of the miR393 overexpression that inhibit apical meristem development observed in Arabidopsis (Wang et al., 2017). Interestingly, the cambium zone, pith, xylem, and phloem regions of the STTM393 lines were wider than the non-transgenic control plants, indicating that STTM393 could promote cell division, resulting in the increase of cell numbers in the pith, xylem, cambium, and phloem, which eventually led to the increase of stem diameter.

Previous studies had shown that miR393 in Arabidopsis and poplar played the role of splicing the target gene, TIR1, and its homologous, FBLs (Vidal et al., 2010; Chen et al., 2011; Tang et al., 2020). In this study, we found that the expression levels of all FBL family members were increased in the STTM393 lines, indicating that the function of miR393 on FBLs was successfully inhibited. A previous study suggested that FBL1-FBL4 in FBL family members could be cleaved by miR393 (Tang et al., 2020). However, in this study, we found that the expression levels of all $F B L$ members were increased using the STTM approach. These results suggest that miR393 may be tolerant to the mismatch with the target sequences. Overexpression of PagFBL1 could promote the growth and root development in poplar (Shu et al., 2018); thus, the promoted secondary growth in STTM393 transgenic plants might be due to the increased expression of $F B L$ family members.

Auxin is a very important hormone in controlling plant growth and development, and the FBL family members as putative auxin receptors mediate the auxin signaling pathway. To further investigate the role of auxin signaling in the development of secondary vascular tissues, we used RNA sequencing to check the genes changed in expression due to the inhibition of miR393. The downstream genes of the auxin signaling pathway, such as SAUR78 and GH3.6, were upregulated, of which GH3.6 was known to be regulated by PagFBL1 and might modulate adventitious rooting (Shu et al., 2018), while the overexpression 
TABLE 1 | The differently expressed genes related to plant growth and wood formation.

\begin{tabular}{|c|c|c|c|c|c|c|c|}
\hline \#ID & $84 K$ & S393-2 & S393-8 & S393-16 & Change & Gene name & Annotation \\
\hline \multicolumn{8}{|c|}{ Auxin signal transduction } \\
\hline Potri.008G066400.1 & 3.81 & 6.46 & 6.84 & 7.00 & Up & $A \cup X 1$ & Auxin transporter-like protein 1 \\
\hline Potri.001G169000.1 & 90.36 & 339.12 & 255.85 & 213.42 & Up & ABP19a & Auxin-binding protein \\
\hline Potri.013G141900.1 & 507.95 & 1108.03 & 840.21 & 863.32 & Up & ABP19a & Auxin-binding protein \\
\hline Potri.004G165500.1 & 2.59 & 5.26 & 6.99 & 4.90 & Up & SAUR1 & Auxin-responsive protein \\
\hline Potri.004G165600.1 & 0.74 & 2.11 & 4.28 & 4.30 & Up & SAUR1 & Auxin-responsive protein \\
\hline Potri.003G071000.1 & 1.32 & 5.92 & 7.58 & 3.07 & Up & SAUR78 & Auxin-responsive protein \\
\hline Potri.001G410400.1 & 4.10 & 7.14 & 8.73 & 8.19 & Up & GH3.6 & Indole-3-acetic acid-amido synthetase \\
\hline Potri.011G129700.1 & 3.64 & 6.38 & 7.91 & 10.50 & Up & GH3.6 & Indole-3-acetic acid-amido synthetase \\
\hline Potri.004G165800.1 & 0.60 & 2.26 & 2.69 & 4.33 & Up & $\mathrm{ARG7}$ & Indole-3-acetic acid-induced protein \\
\hline \multicolumn{8}{|l|}{ Cell expansion } \\
\hline Potri.013G060800.1 & 21.93 & 41.26 & 46.23 & 44.81 & Up & EXPA10 & Expansin-A10 \\
\hline Potri.009G141400.1 & 2.63 & 11.30 & 10.54 & 9.52 & Up & EXPL2 & Expansin-like A2 \\
\hline Potri.002G236200.1 & 16.16 & 37.93 & 54.84 & 32.96 & Up & $\mathrm{XTH} 2$ & Xyloglucan endotransglucosylase/hydrolase 2 \\
\hline Potri.004G021000.1 & 18.89 & 60.70 & 65.11 & 43.95 & Up & XTH8 & Xyloglucan endotransglucosylase/hydrolase 8 \\
\hline Potri.019G125000.1 & 35.85 & 163.80 & 178.76 & 105.74 & Up & XTH9 & Xyloglucan endotransglucosylase/hydrolase 9 \\
\hline Potri.006G071200.1 & 7.73 & 40.09 & 23.95 & 21.20 & Up & XTH23 & Xyloglucan endotransglucosylase/hydrolase 23 \\
\hline Potri.018G095100.2 & 0.05 & 1.31 & 2.34 & 1.57 & Up & XTH23 & Xyloglucan endotransglucosylase/hydrolase 23 \\
\hline Potri.004G132700.1 & 1.22 & 2.01 & 2.15 & 2.70 & Up & GLC11 & Glucan endo-1,3-beta-glucosidase 11 \\
\hline Potri.008G055900.1 & 5.63 & 9.82 & 12.37 & 11.35 & Up & GLC1 & Glucan endo-1,3-beta-glucosidase 1 \\
\hline Potri.008G192600.1 & 0.79 & 2.20 & 3.02 & 2.26 & Up & GATL9 & Probable galacturonosyltransferase-like 9 \\
\hline Potri.010G038300.1 & 5.32 & 17.42 & 16.08 & 11.07 & Up & GATL9 & Probable galacturonosyltransferase-like 9 \\
\hline Potri.009G006500.1 & 21.21 & 43.10 & 45.58 & 28.49 & Up & $\mathrm{IRX7}$ & Glucuronoxylan glucuronosyltransferase \\
\hline \multicolumn{8}{|l|}{ Cell cyclin } \\
\hline Potri.007G005700.1 & 0.72 & 1.71 & 2.75 & 1.63 & Up & CYCD1;1 & Cyclin-D1-1 \\
\hline Potri.009G086700.1 & 18.22 & 30.55 & 33.24 & 24.59 & Up & CYCD1;1 & Cyclin-D1-1 \\
\hline Potri.002G123000.1 & 8.96 & 12.58 & 16.04 & 13.59 & Up & CYCD3;1 & Cyclin-D3-1 \\
\hline Potri.012G115600.1 & 2.71 & 5.59 & 6.22 & 4.26 & Up & CYCP $4 ; 1$ & Cyclin-P4-1 \\
\hline Potri.014G050400.1 & 21.52 & 56.45 & 69.92 & 44.15 & Up & CYCP $4 ; 1$ & Cyclin-P4-1 \\
\hline \multicolumn{8}{|l|}{ Lignin } \\
\hline Potri.002G004500.1 & 9.86 & 17.34 & 14.05 & 12.90 & Up & CCR1 & Cinnamoyl-CoA reductase 1 \\
\hline Potri.013G079500.2 & 2.64 & 9.01 & 9.23 & 6.20 & Up & CCRL6 & Cinnamoyl-CoA reductase-like \\
\hline Potri.014G106500.2 & 1.70 & 3.07 & 2.72 & 3.48 & Up & OMT1 & Caffeic acid 3-O-methyltransferase \\
\hline Potri.007G053400.1 & 9.25 & 18.01 & 17.71 & 12.44 & Up & PER73 & Peroxidase 73 \\
\hline Potri.002G031200.1 & 0.25 & 0.60 & 0.80 & 0.87 & Up & PER9 & Peroxidase 9 \\
\hline Potri.005G028200.1 & 12.56 & 34.36 & 38.49 & 25.00 & Up & BEAT & Acetyl-CoA-benzylalcohol acetyltransferase \\
\hline Potri.019G001400.1 & 2.99 & 6.26 & 7.54 & 5.16 & Up & BEAT & Acetyl-CoA-benzylalcohol acetyltransferase \\
\hline Potri.005G028500.1 & 9.96 & 27.35 & 29.87 & 20.98 & Up & BEAT & Acetyl-CoA-benzylalcohol acetyltransferase \\
\hline \multicolumn{8}{|l|}{ Cellulose } \\
\hline Potri.005G087500.1 & 29.09 & 52.39 & 53.63 & 46.34 & Up & CESA6 & Cellulose synthase 6 \\
\hline Potri.018G029400.1 & 51.33 & 81.41 & 84.90 & 69.36 & Up & CESA1 & Cellulose synthase 1 \\
\hline Potri.003G142300.1 & 18.82 & 9.94 & 10.25 & 10.79 & Down & CSLG3 & Cellulose synthase like G3 \\
\hline Potri.006G004300.1 & 50.81 & 32.43 & 29.52 & 30.59 & Down & CSLE1 & Cellulose synthase like E1 \\
\hline Potri.006G004300.10 & 4.00 & 2.66 & 1.52 & 2.28 & Down & CSLE1 & Cellulose synthase like E1 \\
\hline
\end{tabular}

of SAUR78 promoted plant growth in Arabidopsis (Li et al., 2015). Several cyclin genes, including $C Y C D$ and $C Y C P$, were also upregulated in the STTM393 lines. Cyclin D can regulate cell transformation from the G1 phase to the $S$ phase to start the cell cycle by promoting DNA synthesis and cell proliferation ( $\mathrm{Lu}$ et al., 2011). Overexpression of CYCD2 in tobacco could increase the rate of plant growth and biological yield by accelerating the rate of cell division (Cockcroft et al., 2000). Similarly, CYCD3 was a positive regulator of cambial cell proliferation and secondary growth (Collins et al., 2015). Therefore, the strengthened auxin 

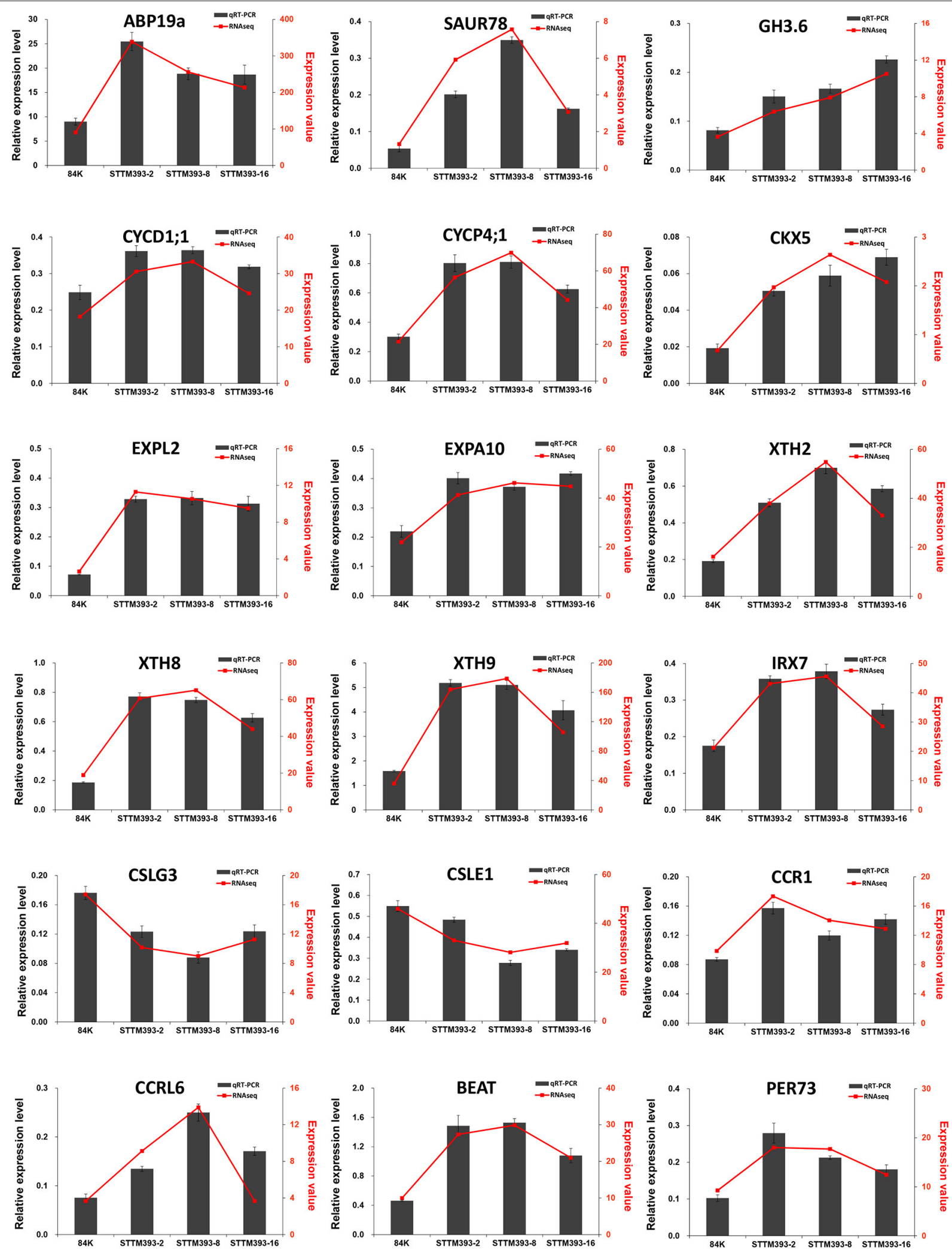

FIGURE 5 | Validation of key genes related to plant growth and wood formation by qRT-PCR using PP2A-2 as reference gene. The column represents the results of qRT-PCR, with the coordinate axis on the left (dark-grey). The line represents the results of transcriptome, with the coordinate axis on the right (red). 
signaling also leads to the activation of cell cycles to accelerate cell division and thus promote the secondary growth of poplar.

Plant cell wall loosening is a necessary physiological process during cell expansion and elongation throughout the entire growth and development of plants. It is due to the hydrolysis of polysaccharides in the cell wall caused by the action of cell wall loosening factors (Zhao and Li, 2011). The expression levels of several cell wall loosening factors, such as EXPA10, $X T H 2, X T H 8, X T H 9$, and XTH23 genes, were upregulated in the transgenic lines. Previous studies have shown that EXPA is involved in the regulation of xylem formation (Gray-Mitsumune et al., 2004), while XTH family members are involved in the cell wall synthesis in poplar (Mellerowicz and Sundberg, 2008), but XTH9 mutation leads to shorter internode length (Hyodo et al., 2003). The upregulation of these genes provides support to the observed fast xylem development in transgenic lines. In addition, the lignin synthesis pathway genes, CCR1, CCRL6, PER73, and BEAT, were upregulated in the STTM393 lines, in comparison with the control plants. The increased expression of key enzymes in the lignin synthesis pathway can promote lignin synthesis (Xie et al., 2018). On the contrary, the expression levels of two cellulose synthase-related genes, CSLG3 and CSLE1, in STTM393 lines were decreased. This was in accordance with the observation that STTM393 lines exhibited higher lignin content but lower cellulose content in stems.

In summary, the STTM approach can block the function of miR393, thus increasing the expression of its target genes FBLs. The strengthened auxin signaling promotes secondary growth of and biomass production in transgenic poplars, by changing a series of downstream genes orchestrating cambium activity, xylem development, and cell wall synthesis. This study provides additional support, that is, manipulating auxin signaling regulators could enhance trees for fast growth and biomass production.

\section{REFERENCES}

Bartel, D. P. (2009). MicroRNAs: target recognition and regulatory functions. Cell 136, 215-233. doi: 10.1016/j.cell.2009.01.002

Bian, H., Xie, Y., Guo, F., Han, N., Ma, S., Zeng, Z., et al. (2012). Distinctive expression patterns and roles of the miRNA393/TIR1 homolog module in regulating flag leaf inclination and primary and crown root growth in rice (Oryza sativa). New Phytol. 196, 149-161. doi: 10.1111/j.1469-8137.2012.04248.x

Busov, V. (2018). Manipulation of growth and architectural characteristics in trees for increased woody biomass production. Front. Plant Sci. 9:1505. doi: 10.3389/fpls.2018.01505

Cao, D., Wang, J., Ju, Z., Liu, Q., Li, S., Tian, H., et al. (2016). Regulations on growth and development in tomato cotyledon, flower and fruit via destruction of miR396 with short tandem target mimic. Plant Science. 247, 1-12. doi: 10.1016/j.plantsci.2016.02.012

Chen, Z.-H., Bao, M.-L., Sun, Y.-Z., Yang, Y.-J., Xu, X.-H., Wang, J.H., et al. (2011). Regulation of auxin response by miR393-targeted transport inhibitor response protein 1 is involved in normal development in Arabidopsis. Plant Mol. Biol. 77, 619-629. doi: 10.1007/s11103-0119838-1

\section{DATA AVAILABILITY STATEMENT}

The datasets presented in this study can be found in online repositories. The names of the repository/repositories and accession number(s) can be found below: SRA, PRJNA724789.

\section{AUTHOR CONTRIBUTIONS}

FT designed the study. FT constructed the STTM393 vector and LC transformed it into poplar. FT, $\mathrm{XH}$, and LC investigated the growth data of STTM393 transgenic and control plants. FT and $\mathrm{XH}$ completed the section and microscopic observation of different stem nodes of STTM393 plants. FT and LC analyzed the data and drafted the manuscript. WS and LW helped in the interpretation of results. All of the authors carefully checked and approved this manuscript.

\section{FUNDING}

This work was supported by the National Natural Science Foundation of China (31700592), the Fundamental Research Funds for the Central Non-profit Research Institution of CAF (CAFYBB2017QA003), and the National Key Research and Development Program of China (2016YFD0600100).

\section{ACKNOWLEDGMENTS}

We thank Zhongyong Chen and Jun'e Mu of the Chinese Academy of Forestry for their valuable technical contribution for the transformation and tissue culture of poplar.

\section{SUPPLEMENTARY MATERIAL}

The Supplementary Material for this article can be found online at: https://www.frontiersin.org/articles/10.3389/fpls.2021. 714907/full\#supplementary-material

Cockcroft, C., den Boer, B., Healy, S., and Murray, J. (2000). Cyclin D control of growth rate in plants. Nature 405, 575-579. doi: 10.1038/35014621

Collins, C., Gowda, M., and Jahn, C. (2015). CYCD3 D-type cyclins regulate cambial cell proliferation and secondary growth in Arabidopsis. J. Exp. Bot. 66, 4595-4606. doi: 10.1093/jxb/erv218

Curaba, J., Talbot, M., Li, Z., and Helliwell, C. (2013). Over-expression of microRNA171 affects phase transitions and floral meristem determinancy in barley. BMC Plant Biol. 13:6. doi: 10.1186/1471-2229-13-6

Gao, P., Bai, X., Yang, L., Lv, D., Pan, X., Li, Y., et al. (2011). Osa-MIR393: a salinity- and alkaline stress-related microRNA gene. Mol. Biol. Rep. 38, 237-242. doi: 10.1007/s11033-010-0100-8

Gray-Mitsumune, M., Mellerowicz, E., Abe, H., Schrader, J., Winzéll, A., Sterky, F., et al. (2004). Expansins abundant in secondary xylem belong to subgroup A of the -expansin gene family. Plant Physiol. 135, 1552-1564. doi: 10.1104/pp.104.039321

Gu, Z., Huang, C., Li, F., and Zhou, X. (2014). A versatile system for functional analysis of genes and microRNAs in cotton. Plant Biotechnol. J. 12, 638-649. doi: $10.1111 /$ pbi.12169

Gui, J., Lam, P. Y., Tobimatsu, Y., Sun, J., Huang, C., Cao, S., et al. (2020). Fiberspecific regulation of lignin biosynthesis improves biomass quality in Populus. New Phytol. 226, 1074-1087. doi: 10.1111/nph.16411 
Guo, Z., Kuang, Z., Wang, Y., Zhao, Y., Tao, Y., Cheng, C., et al. (2019). PmiREN: a comprehensive encyclopedia of plant miRNAs. Nucleic Acids Res. 48, D1114D1121. doi: 10.1093/nar/gkz894

Hu, Z., Keceli, M., Kalliola, M., Li, J., Survila, M., Heino, P., et al. (2012). F-box protein AFB4 plays a crucial role in plant growth, development and innate immunity. Cell Res. 22, 777-781. doi: 10.1038/cr.2012.12

Hyodo, H., Yamakawa, S., Takeda, Y., Tsuduki, M., Yokota, A., Nishitani, K., et al. (2003). Active gene expression of a xyloglucan endotransglucosylase/hydrolase gene, XTH9, in inflorescence apices is related to cell elongation in Arabidopsis thaliana. Plant Mol. Biol. 52, 473-482. doi: 10.1023/A:1023904217641

Li, Z., Chen, H., Li, Q., Tao, J., Bian, X.-H., Ma, B., et al. (2015). Three SAUR proteins SAUR76, SAUR77 and SAUR78 promote plant growth in Arabidopsis. Sci. Rep. 5:12477. doi: 10.1038/srep 12477

Liu, X., Liu, S., Wang, R., Chen, X., Fan, Z., Wu, B., et al. (2019). Analyses of miRNA functions in maize using a newly developed ZMBJ-CMV-2bN81STTM vector. Front. Plant Sci. 10:1277. doi: 10.3389/fpls.2019.01277

Lu, M., Yao, C., Lai, D., and Xu, H. (2011). Cyclin D1 and cell cycle regulation. Biotechnol. Bull. 10, 55-59. doi: 10.13560/j.cnki.biotech.bull.1985.2011.10.025

Lu, S., Sun, Y.-H., and Chiang, V. L. (2008). Stress-responsive microRNAs in Populus. Plant J. 55, 131-151. doi: 10.1111/j.1365-313X.2008.03497.x

Lucas, W. J., Groover, A., Lichtenberger, R., Furuta, K., Shri-Ram, Y., Helariutta, Y., et al. (2013). The plant vascular system: evolution, development and functions. J. Integr. Plant Biol. 55, 294-388. doi: 10.1111/jipb.12041

Mellerowicz, E., and Sundberg, B. (2008). Wood cell walls: biosynthesis, developmental dynamics and their implications for wood properties. Curr. Opin. Plant Biol. 11, 293-300. doi: 10.1016/j.pbi.2008.03.003

Meyers, B. C., Axtell, M. J., Bartel, B., Bartel, D. P., Baulcombe, D., Bowman, J. L., et al. (2008). Criteria for annotation of plant MicroRNAs. Plant Cell 20, 3186-3190. doi: 10.1105/tpc.108.064311

Navarro, L., Dunoyer, P., Jay, F., Arnold, B., Dharmasiri, N., Estelle, M., et al. (2006). A plant miRNA contributes to antibacterial resistance by repressing auxin signaling. Science 312, 436-439. doi: 10.1126/science.1126088

Parry, G., Calderon-Villalobos, L. I., Prigge, M., Peret, B., Dharmasiri, S., Itoh, H., et al. (2009). Complex regulation of the TIR1/AFB family of auxin receptors. Proc. Natl. Acad. Sci. U. S. A. 106, 22540-22545. doi: 10.1073/pnas.0911967106

Peng, T., Qiao, M., Liu, H., Teotia, S., Zhang, Z., Zhao, Y., et al. (2018). A resource for inactivation of microRNAs using short tandem target mimic technology in model and crop plants. Mol. Plant 11, 1400-1417. doi: 10.1016/j.molp.2018.09.003

Sha, A., Zhao, J., Yin, K., Tang, Y., Wang, Y., Wei, X., et al. (2014). Virus-based microRNA silencing in plants. Plant Physiol. 164, 36-47. doi: 10.1104/pp.113.231100

Shu, W., Liu, Y., Guo, Y., Zhou, H., Zhang, J., Zhao, S., et al. (2015). A Populus TIR1 gene family survey reveals differential expression patterns and responses to 1-naphthaleneacetic acid and stress treatments. Front. Plant Sci. 6:714. doi: $10.3389 /$ fpls.2015.00719

Shu, W., Zhou, H., Jiang, C., Zhao, S., Wang, L., Li, Q., et al. (2018). The auxin receptor TIR1 homolog (PagFBL1) regulates adventitious rooting through interactions with Aux/IAA28 in Populus. Plant Biotechnol. J. 17, 338-349. doi: $10.1111 /$ pbi. 12980

Si-Ammour, A., Windels, D., Arn-Bouldoires, E., Kutter, C., Ailhas, J., Meins, F., et al. (2011). miR393 and secondary siRNAs regulate expression of the TIR1/AFB2 auxin receptor clade and auxin-related development of Arabidopsis leaves. Plant Physiol. 157, 683-691. doi: 10.1104/pp.111.180083

Sieber, P., Wellmer, F., Gheyselinck, J., Riechmann, J., and Meyerowitz, E. (2007). Redundancy and specialization among plant microRNAs: role of the MIR164 family in developmental robustness. Development 134, 1051-1060. doi: 10.1242/dev.02817

Sundell, D., Street, N. R., Kumar, M., Mellerowicz, E. J., Kucukoglu, M., Johnsson, C., et al. (2017). AspWood: high-spatial-resolution transcriptome profiles reveal uncharacterized modularity of wood formation in Populus tremula. Plant Cell 29, 1585-1604. doi: 10.1105/tpc.17.00153

Tang, F., Chu, L., He, X., Shu, W., and Lu, M. (2020). Identification of regulation of poplar miR393 on FBL family genes. J. Central South Univ. Forest. Technol. 40, 146-153. doi: 10.14067/j.cnki.1673-923x.2020.05.017
Tang, F., Chu, L., Shu, W., He, X., Wang, L., and Lu, M. (2019). Selection and validation of reference genes for quantitative expression analysis of miRNAs and mRNAs in Poplar. Plant Methods 15:35. doi: 10.1186/s13007-019-0420-1

Tang, F., Wei, H., Zhao, S., Wang, L., Zheng, H., and Lu, M. (2016). Identification of microRNAs Involved in Regeneration of the Secondary Vascular System in Populus tomentosa Carr. Front. Plant Sci. 7:724. doi: 10.3389/fpls.2016. 00724

Tang, G., Yan, J., Gu, Y., Qiao, M., Fan, R., Mao, Y., et al. (2012). Construction of short tandem target mimic (STTM) to block the functions of plant and animal microRNAs. Methods 58, 118-125. doi: 10.1016/j.ymeth.2012.10.006

Teotia, S., Zhang, D., and Tang, G. (2017). Knockdown of rice microRNA166 by short tandem target mimic (STTM). Methods Mol. Biol. 1654, 337-349. doi: 10.1007/978-1-4939-7231-9_25

Vidal, E., Araus, V., Lu, C., Parry, G., Green, P., Coruzzi, G., et al. (2010). Nitrateresponsive miR393/AFB3 regulatory module controls root system architecture in Arabidopsis thaliana. Proc. Natl. Acad. Sci. U. S. A. 107, 4477-4482. doi: 10.1073/pnas.0909571107

Voinnet, O. (2009). Origin, biogenesis, and activity of plant MicroRNAs. Cell 136, 669-687. doi: 10.1016/j.cell.2009.01.046

Wang, F., Shi, D., Han, J., Zhang, G., Jiang, X., Yang, M., et al. (2020). Comparative study on pretreatment processes for different utilization purposes of switchgrass. ACS Omega 5, 21999-22007. doi: 10.1021/acsomega.0 c01047

Wang, L., Liu, Z., Qiao, M., and Xiang, F. (2017). miR393 inhibits in vitro shoot regeneration in Arabidopsis thaliana via repressing TIR1. Plant Sci. 266, 1-8. doi: $10.1016 /$ j.plantsci.2017.10.009

Wójcik, A., and Gaj, M. (2016). miR393 contributes to the embryogenic transition induced in vitro in Arabidopsis via the modification of the tissue sensitivity to auxin treatment. Planta 244, 231-243. doi: 10.1007/s00425-016-2505-7

Wong, J., Gao, l., Yang, Y., Zhai, J., Arikit, S., Yu, Y., et al. (2014). Roles of small RNAs in soybean defense against Phytophthora sojae infection. Plant J. 79, 928-940. doi: 10.1111/tpj.12590

Xie, M., Zhang, J., Tschaplinski, T., Tuskan, G., Chen, J.-G., and Muchero, W. (2018). Regulation of lignin biosynthesis and its role in growth-defense tradeoffs. Front. Plant Sci. 9:1427. doi: 10.3389/fpls.2018.01427

Yan, J., Gu, Y., Jia, X., Kang, W., Pan, S., Tang, X., et al. (2012). Effective small RNA destruction by the expression of a short tandem target mimic in Arabidopsis. Plant Cell 24, 415-427. doi: 10.1105/tpc.111.094144

Yuan, W., Suo, J., Shi, B., Zhou, C., Bai, B., Bian, H., et al. (2019). The barley miR393 has multiple roles in regulation of seedling growth, stomatal density, and drought stress tolerance. Plant Physiol. Biochem. 142, 303-311. doi: 10.1016/j.plaphy.2019.07.021

Zhang, B., Pan, X., Cannon, C., Cobb, G., and Anderson, T. (2006). Conservation and divergence of plant microRNA genes. Plant J. 46, 243-259. doi: 10.1111/j.1365-313X.2006.02697.x

Zhang, X., Zou, Z., Gong, P., Zhang, J., Ziaf, K., Li, H., et al. (2010). Overexpression of microRNA169 confers enhanced drought tolerance to tomato. Biotechnol. Lett. 33, 403-409. doi: 10.1007/s10529-010-0436-0

Zhao, Y., and Li, L. (2011). Plant cell wall loosening factors. Plant Physiol. J. 47, 925-935. doi: 10.13592/j.cnki.ppj.2011.10.008

Zhu, Q.-H., Upadhyaya, N., Gubler, F., and Helliwell, C. (2009). Over-expression of miR172 causes loss of spikelet determinacy and floral organ abnormalities in rice (Oryza sativa). BMC Plant Biol. 9:149. doi: 10.1186/1471-2229-9-149

Conflict of Interest: The authors declare that the research was conducted in the absence of any commercial or financial relationships that could be construed as a potential conflict of interest.

Copyright (c) $2021 \mathrm{Chu}, \mathrm{He}$, Shu, Wang and Tang. This is an open-access article distributed under the terms of the Creative Commons Attribution License (CC BY). The use, distribution or reproduction in other forums is permitted, provided the original author(s) and the copyright owner(s) are credited and that the original publication in this journal is cited, in accordance with accepted academic practice. No use, distribution or reproduction is permitted which does not comply with these terms. 